\title{
Thermodynamic Nonequilibrium Features in Binary Diffusion*
}

\author{
Chuan-Dong Lin (林传东), ${ }^{1,2, \dagger}$ Kai-Hong Luo (罗开红), ${ }^{1,3, \ddagger}$ Yan-Biao Gan (甘延标), ${ }^{2,4}$ and \\ Hui-Lin Lai (赖惠林) ${ }^{2}$ \\ ${ }^{1}$ Center for Combustion Energy, Key Laboratory for Thermal Science and Power Engineering of Ministry of Education, \\ Department of Energy and Power Engineering, Tsinghua University, Beijing 100084, China \\ ${ }^{2}$ College of Mathematics and Informatics \& FJKLMAA, Fujian Normal University, Fuzhou 350007, China \\ ${ }^{3}$ Department of Mechanical Engineering, University College London, Torrington Place, London WC1E 7JE, UK \\ ${ }^{4}$ North China Institute of Aerospace Engineering, Langfang 065000, China
}

(Received January 8, 2018; revised manuscript received February 26, 2018)

\begin{abstract}
Diffusion is a ubiquitous physical phenomenon where thermodynamic nonequilibrium effects (TNEs) are outstanding issues. In this work, we employ the discrete Boltzmann method to investigate the TNEs in the dynamic process of binary diffusion. The main features of the distribution function in velocity space are recovered and discussed. It is found that, with the decreasing gradients of macroscopic quantities (such as density, concentration, velocity, etc.), both the local and global TNEs decrease with the time but increase with the relaxation time in a power law, respectively.
\end{abstract}

PACS numbers: 05.20.Dd, 05.70.Ln, 51.20.+d, 52.25.Fi

Key words: ...

Diffusion is the net movement of particles down their concentration gradient. It takes place when miscible materials are brought together. ${ }^{[1]}$ It is a fundamental and ubiquitous phenomenon in nature and widely exists in engineering, sciences, technologies and beyond.. ${ }^{[1]}$ For example, diffusion plays a key role in the storage and mining of shale gas, partially premixed or nonpremixed combustion, etc. Physically, diffusion is a typical scenario that incorporates various essential thermodynamic nonequilibrium effects (TNEs). Early in 1855, Fick's laws of diffusion were presented in the background of thermodynamics and nonequilibrium thermodynamics. ${ }^{[1]}$ Later, the theory of diffusion in gases was developed based on Boltzmann's equation. ${ }^{[2]}$ However, the nonlinear complexity and pronounced TNEs often provide challenges to previous theoretical studies.

In recent decades, researches into nonlinear complex systems have been significantly promoted by numerical approaches, such as the lattice Boltzmann method (LBM). ${ }^{[3-13]}$ In 2004, Briant and Yeomans utilized the LBM to simulate the relative diffusion of two fluid components in the vicinity of the contact line. ${ }^{[4]}$ In 2016, an LBM was developed for solving the fractional advection-diffusion equation. ${ }^{[10]}$ However, previous LBMs were mainly used to solve hydrodynamic equations, but ignored some detained TNEs. As a variant of traditional
LBMs, the discrete Boltzmann method (DBM) ${ }^{[14-18]}$ has achieved great success in simulating nonequilibrium systems, such as multiphase flows, fluid instabilities, combustion, etc. Very recently, the TNEs in binary diffusion were demonstrated by a double-distribution-function DBM. [17]

In this work, we adopt the DBM to have a deeper probe into the nonequilibrium features of binary diffusion. The $\mathrm{DBM}$ is based on the following equation, ${ }^{[17]}$

$$
\frac{\partial f_{i}^{\sigma}}{\partial t}+\boldsymbol{v}_{i}^{\sigma} \cdot \nabla f_{i}^{\sigma}=-\frac{1}{\tau}\left(f_{i}^{\sigma}-f_{i}^{\sigma \mathrm{eq}}\right),
$$

where the superscript $\sigma$ denotes species, $t$ is the time, $\tau$ is the relaxation time, $\boldsymbol{v}_{i}^{\sigma}$ indicates the discrete velocity, $f_{i}^{\sigma}$ is the discrete distribution function, $f_{i}^{\sigma \mathrm{eq}}=f_{i}^{\sigma \mathrm{eq}}\left(n^{\sigma}, \boldsymbol{u}, T\right)$ is the discrete equilibrium distribution function that depends upon the molar density $n^{\sigma}$, the mixture hydrodynamic velocity $\boldsymbol{u}$, and the mixture temperature $T$. The expression of $f_{i}^{\sigma \text { eq }}$ refers to Ref. [19]. It has been demonstrated that the DBM could not only recover the modified Navier-Stokes equations for binary fluid system but also conveniently capture the following nonequilibrium quantities,

$$
\begin{aligned}
& \boldsymbol{\Delta}_{2}^{\sigma *}=\sum_{i}\left(f_{i}^{\sigma}-f_{i}^{\sigma \mathrm{eq} *}\right) \boldsymbol{v}_{i}^{\sigma *} \boldsymbol{v}_{i}^{\sigma *} \\
& \boldsymbol{\Delta}_{3,1}^{\sigma *}=\sum_{i}\left(f_{i}^{\sigma}-f_{i}^{\sigma \mathrm{eq} *}\right)\left(\boldsymbol{v}_{i}^{\sigma *} \cdot \boldsymbol{v}_{i}^{\sigma *}+\eta_{i}^{\sigma 2}\right) \boldsymbol{v}_{i}^{\sigma *},
\end{aligned}
$$

\footnotetext{
*Supported by the MOST National Key Research and Development Programme under Grant No. 2016YFB0600805, the China Postdoctoral Science Foundation under Grant No. 2017M620757, the Center for Combustion Energy at Tsinghua University, Natural Science Foundation of Hebei Province under Grant Nos. A2017409014, ZD2017001 and A201500111, FJKLMAA, Fujian Normal University, and the UK Engineering and Physical Sciences Research Council under the Project UK Consortium on Mesoscale Engineering Sciences (UKCOMES) under Grant No. EP/L00030X/1 is gratefully acknowledged

${ }^{\dagger}$ E-mail: chuandonglin@163.com

${ }_{\ddagger}^{\ddagger}$ E-mail: K.Luo@ucl.ac.uk
} 


$$
\begin{aligned}
& \boldsymbol{\Delta}_{3}^{\sigma *}=\sum_{i}\left(f_{i}^{\sigma}-f_{i}^{\sigma \mathrm{eq} *}\right) \boldsymbol{v}_{i}^{\sigma *} \boldsymbol{v}_{i}^{\sigma *} \boldsymbol{v}_{i}^{\sigma *}, \\
& \boldsymbol{\Delta}_{4,2}^{\sigma *}=\sum_{i}\left(f_{i}^{\sigma}-f_{i}^{\sigma \mathrm{eq} *}\right)\left(\boldsymbol{v}_{i}^{\sigma *} \cdot \boldsymbol{v}_{i}^{\sigma *}+\eta_{i}^{\sigma 2}\right) \boldsymbol{v}_{i}^{\sigma *} \boldsymbol{v}_{i}^{\sigma *},
\end{aligned}
$$

with $f_{i}^{\sigma \text { eq* }}=f_{i}^{\sigma \text { eq }}\left(n^{\sigma}, \boldsymbol{u}^{\sigma}, T^{\sigma}\right)$ and the central velocity $\boldsymbol{v}_{i}^{\sigma *}=\boldsymbol{v}_{i}^{\sigma}-\boldsymbol{u}^{\sigma}$. Physically, the second-order tensor $\boldsymbol{\Delta}_{2}^{\sigma *}$ is related to the viscous stress tensor and nonorganised momentum fluxes. The vector $\boldsymbol{\Delta}_{3,1}^{\sigma *}$ and the third-order tensor $\boldsymbol{\Delta}_{3}^{\sigma *}$ refer to the nonorganised heat fluxes. The second-order tensor $\boldsymbol{\Delta}_{4,2}^{\sigma *}$ is relevant to the flux of nonorganised heat flux. ${ }^{[18]}$

To conduct numerical simulations, the temporal derivative in Eq. (1) is calculated in its analytical form, ${ }^{[16]}$ and the spatial derivative is solved by the second order non-oscillatory and non-free-parameter dissipation difference scheme. ${ }^{[20]}$ Next, let us investigate the nonequilibrium effects in the binary diffusion. The initial configura-

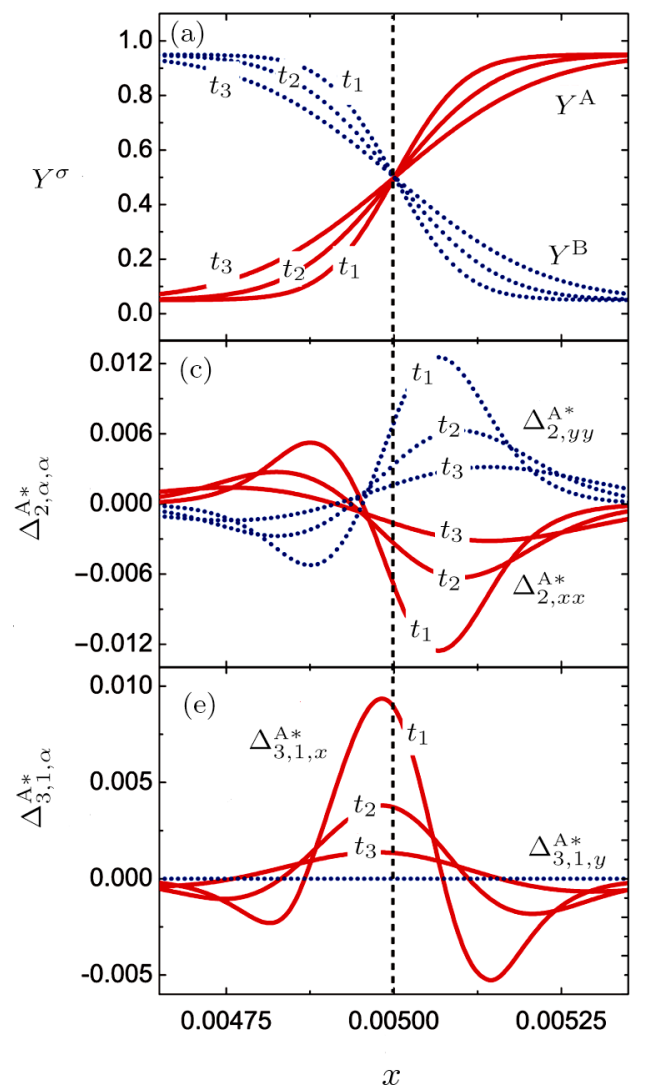

tion is

$$
\begin{aligned}
& \left(Y^{\mathrm{A}}, Y^{\mathrm{B}}\right)_{L}=\left(1-Y_{0}, Y_{0}\right), \\
& \left(Y^{\mathrm{A}}, Y^{\mathrm{B}}\right)_{R}=\left(Y_{0}, 1-Y_{0}\right),
\end{aligned}
$$

where $L(R)$ indicates the left (right) part of the computational domain, and $(\rho, \boldsymbol{u}, T)=\left(1,0, T_{0}\right)$ in the two parts. $Y^{\mathrm{A}}$ and $Y^{\mathrm{B}}$ represent the mole fraction of species $\mathrm{A}$ and $\mathrm{B}$, respectively. Furthermore, the inflow and periodic boundary conditions are adopted in the $x$ and $y$ directions, respectively. First of all, two cases of simulations are performed. In Case I, the initial temperature is $T_{0}=1$, the mole fraction $Y_{0}=5 \%$, the relaxation time $\tau=2 \times 10^{-5}$, the time step $\Delta t=10^{-7}$, the space step $\Delta x=\Delta y=2 \times 10^{-6}$, the mesh $N_{x}=N_{y}=5000 \times 1$. In Case II, the parameters are $T_{0}=2, Y_{0}=25 \%$, $\tau=4 \times 10^{-5}, \Delta t=2 \times 10^{-7}, \Delta x=\Delta y=4 \times 10^{-6}$, $N_{x}=N_{y}=2500 \times 1$.

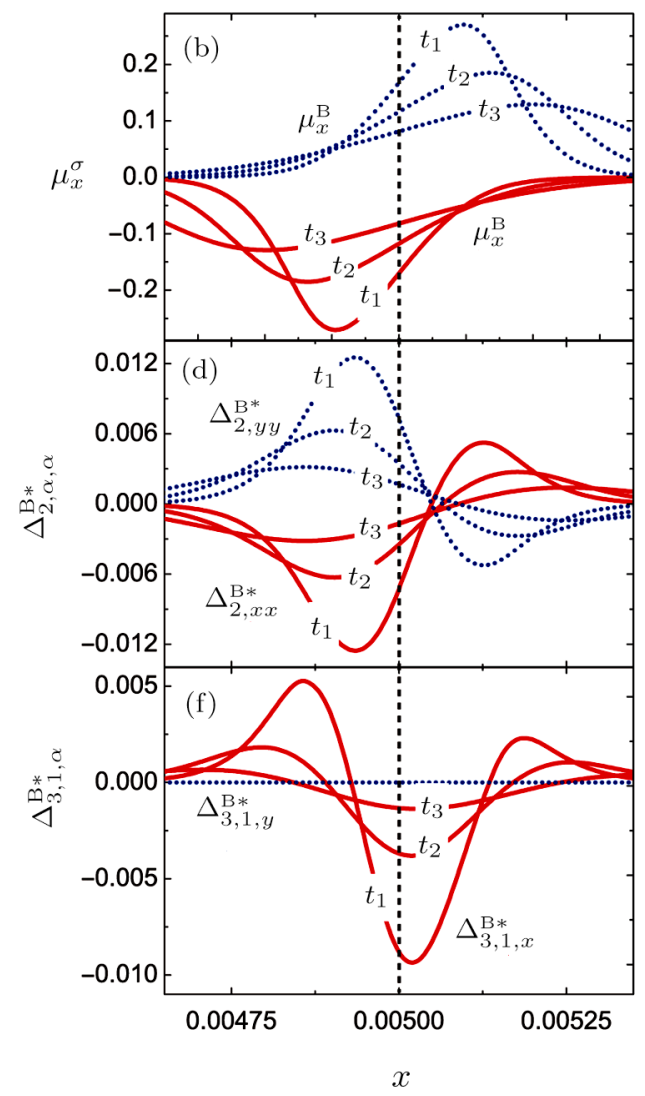

Fig. 1 Physical quantities and TNE characterizations around the material interface at representative instants, $t_{1}=2 \times 10^{-4}, t_{2}=4 \times 10^{-4}$, and $t_{3}=8 \times 10^{-4}$, respectively. (a) Mole fraction $Y^{\sigma}$, (b) horizontal velocity $u_{x}^{\sigma}$, (c) nonorganised energy $\Delta_{2, \alpha \alpha}^{\mathrm{A} *}$, (d) nonorganised energy $\Delta_{2, \alpha \alpha}^{\mathrm{B} *}$, (e) nonorganised energy flux $\Delta_{3,1, \alpha}^{\mathrm{A} *}$, and (f) nonorganised energy flux $\Delta_{3,1, \alpha}^{\mathrm{B} *}$. In each plot, a vertical line is plotted at the material interface.

Figure 1 delineates the physical quantities around the material interface in Case I at time instants, $t_{1}=2 \times 10^{-4}$, $t_{2}=4 \times 10^{-4}$, and $t_{3}=8 \times 10^{-4}$, respectively. The behaviors in Case II are similar to those in Case I (not shown here). Figures $1(\mathrm{a})-1(\mathrm{~b})$ show that the gradients of phys- ical quantities $Y^{\sigma}$ and $u_{x}^{\sigma}$ become smooth with time, the trough of $u_{x}^{\mathrm{A}}$ and the peak of $u_{x}^{\mathrm{B}}$ move away from the interface. Meanwhile, as shown in Figs. 1(c)-1(f), the nonequilibrium physical quantities $\Delta_{2, \alpha \alpha}^{\sigma *}$ and $\Delta_{3,1, \alpha}^{\sigma *}$ reduce because the TNEs depend on physical gradients. ${ }^{[17]}$ 
Moreover, the TNEs of the two species are contrary due to the opposite physical gradients of them, and they have different tendencies. Specifically, $\Delta_{2, \alpha \alpha}^{\sigma *}$ has a trough and a peak, while $\Delta_{3,1, x}^{\sigma *}$ has two troughs or two peaks. The troughs or peaks of them do not coincide and depart from the interface due to the moving trough and peak of $Y^{\sigma}$ and $u_{x}^{\sigma}$. In addition, $\Delta_{2, x x}^{\sigma *}+\Delta_{2, y y}^{\sigma *}=0$ due to the conservation of energy, and $\Delta_{3,1, y}^{\sigma *}=0$ because there is no energy flux in the $y$ direction.

In fact, we can recover the main characteristics of the distribution function $f^{\sigma}$ from the above nonequilibrium physical quantities. Now, let us analyze these nonequilib- rium manifestations at the material interface labeled by the guideline in Fig. 1. Clearly, $\Delta_{2, x x}^{A *}<0$ indicates that $f^{\mathrm{A}}\left(v_{x}\right)$ is thinner and higher than its equilibrium counterpart $f^{\text {Aeq }}\left(v_{x}\right)$, and $\Delta_{2, y y}^{A *}>0$ means that $f^{\mathrm{A}}\left(v_{y}\right)$ is fatter and lower than its equilibrium counterpart $f^{\text {Aeq }}\left(v_{y}\right)$. Meanwhile, $\Delta_{3,1, x}^{A *}>0$ indicates that the portion of $f^{\mathrm{A}}\left(v_{x}\right)$ for $v_{x}>0$ is larger than its portion for $v_{x}<0$, and $\Delta_{3,1, y}^{A *}=0$ because $f^{\mathrm{A}}\left(v_{y}\right)$ is symmetric about the axis $v_{y}=u_{y}^{\mathrm{A}}=0$. The sketch of $f^{\mathrm{A}}$ versus $v_{x}$ or $v_{y}$ is plotted in Fig. 2(a), and the main feature of $f^{\mathrm{A}}$ in the velocity space $\left(v_{x}, v_{y}\right)$ is shown in Fig. 2(c). Similarly, the sketch of $f^{\mathrm{B}}$ is plotted in Figs. 2(b) and 2(d).
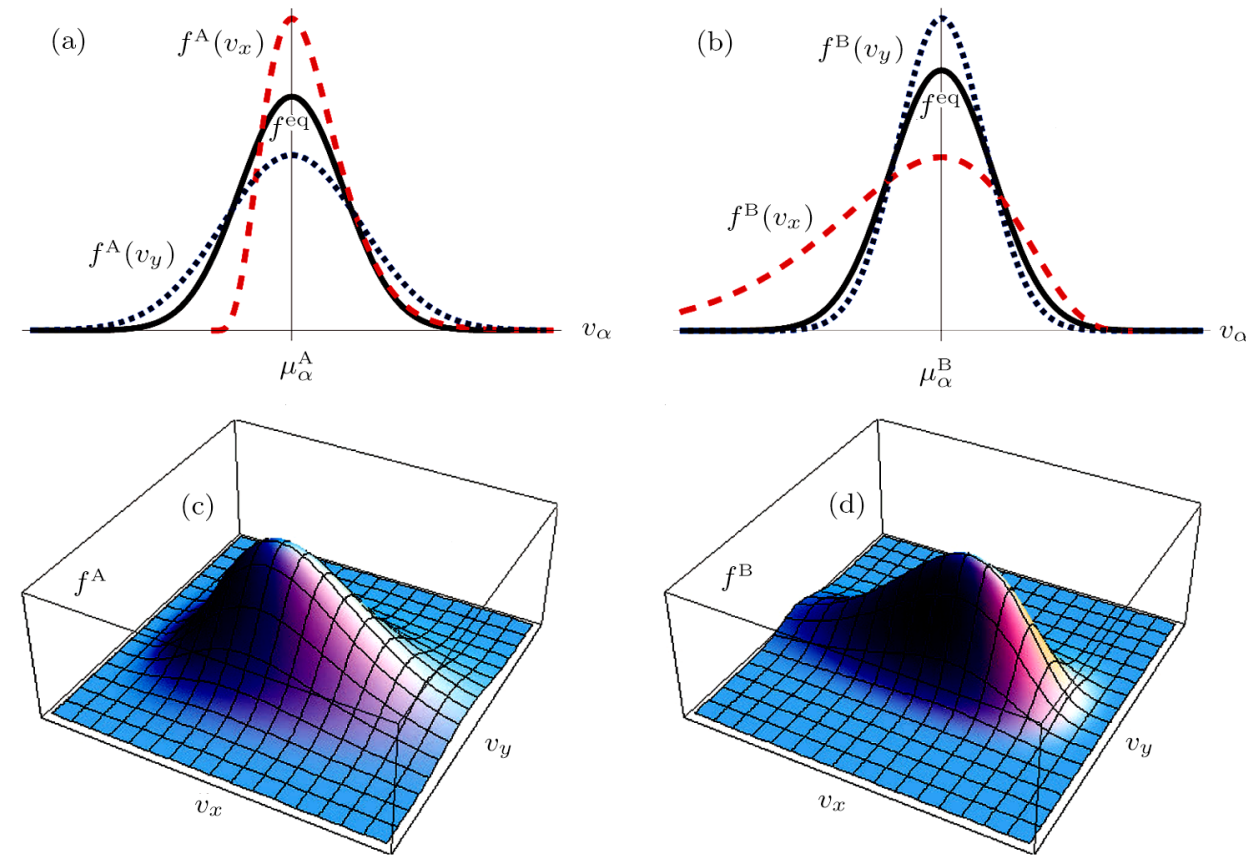

Fig. 2 Sketches of the velocity distribution functions at the material interface: (a) $f^{\mathrm{A}}$ versus $v_{x}$ or $v_{y}$, (b) $f^{\mathrm{B}}$ versus $v_{x}$ or $v_{y},(\mathrm{c}) f^{\mathrm{A}}$ in the velocity space $\left(v_{x}, v_{y}\right)$, and (d) $f^{\mathrm{B}}$ in the velocity space $\left(v_{x}, v_{y}\right)$.

In order to obtain a better understanding of the TNEs, let us monitor the evolution of those nonequilibrium quantities in the process of the binary diffusion. Figure 3(a) illustrates the amplitude and integral of $\Delta_{2, x x}^{\sigma}$ versus time in Case I. Here the amplitude, $\left|\Delta_{2, x x}^{\sigma}\right| \max$, is defined as the half distance between the peak and trough of $\Delta_{2, x x}^{\sigma}$ near the material interface, and the integral, $\iint\left|\Delta_{2, x x}^{\sigma}\right| \mathrm{d} x \mathrm{~d} y$, is extended over the region, $0 \leq x \leq 0.01$ and $0 \leq y \leq 1$. Figure $3(\mathrm{~b})$ is for $\Delta_{3,1, x}^{\sigma}$ versus time in Case I. Figures 3(c)-3(d) are for Case II. Squares and triangles stand for the species $\mathrm{A}$ and $\mathrm{B}$, respectively. Lines denote the fitting functions, $F(\phi)=-13.2-0.997 \phi$ and $F(\phi)=-17.0-0.499 \phi$ in Fig. $3(\mathrm{a}), F(\phi)=-17.5-1.49 \phi$ and $F(\phi)=-21.4-0.984 \phi$ in Fig. $3(\mathrm{~b}), F(\phi)=-12.3-\phi$ and $F(\phi)=-15.4-\phi$ in Fig. $3(\mathrm{c}), F(\phi)=-15.8-1.45 \phi$ and $F(\phi)=-21.4-0.984 \phi$ in Fig. $3(\mathrm{~d})$, respectively, with $\phi=\ln (t)$. In fact, the amplitudes of $\Delta_{2, x x}^{\sigma}$ and $\Delta_{3,1, x}^{\sigma}$ represent the local strongest TNEs, while $\iint\left|\Delta_{2, x x}^{\sigma}\right| \mathrm{d} x \mathrm{~d} y$ and $\iint \mid \Delta_{3,1, x}^{\sigma} \mathrm{d} x \mathrm{~d} y$ indicate the global TNEs in the physical region. It can be found that, both the local and global TNEs become weaker with time due to the decrease of gradient forces, and the relationships between these TNEs and the time satisfy a power law.

To investigate the dependence of the TNEs on the relaxation time, we carry out two groups of simulations. Group I has $T_{0}=1$ and $Y_{0}=5 \%$. Group II has $T_{0}=2$ and $Y_{0}=25 \%$. Considering both the accuracy and efficiency, we choose the time and space steps as $(\Delta t, \Delta x)=$ $\left(5 \times 10^{-8}, 10^{-6}\right),\left(10^{-7}, 2 \times 10^{-6}\right),\left(2 \times 10^{-7}, 4 \times 10^{-6}\right)$, $\left(4 \times 10^{-7}, 8 \times 10^{-6}\right),\left(8 \times 10^{-7}, 1.6 \times 10^{-5}\right)$ for simula- 
tions with relaxation time $\tau=10^{-5}, 2 \times 10^{-5}, 4 \times 10^{-5}$, $8 \times 10^{-5}, 1.6 \times 10^{-4}$, respectively. Figure 4 plots the relationship between the TNEs and the relaxation time at $t=0.002$. The fitting functions are $F(\phi)=3.71+0.989 \phi$ and $F(\phi)=2.29+1.50 \phi$ in Fig. $4(\mathrm{a}), F(\phi)=7.69+1.47 \phi$ and $F(\phi)=6.12+1.97 \phi$ in Fig. $4(\mathrm{~b}), F(\phi)=4.02+0.995 \phi$ and $F(\phi)=2.83+1.50 \phi$ in Fig. $4(\mathrm{c}), F(\phi)=7.81+1.44 \phi$ and $F(\phi)=6.66+1.95 \phi$ in Fig. $4(\mathrm{~d})$, respectively, with $\phi=\ln (\tau)$. It is clear that both the local and global TNEs increase with the relaxation time in a power-law form. Theoretically, the physical gradients reduce fast for large relaxation time. The reducing physical gradients and increasing relaxation time exert opposite effects on the TNEs, ${ }^{[17]}$ but the latter dominates for parameter space considered in our simulations.
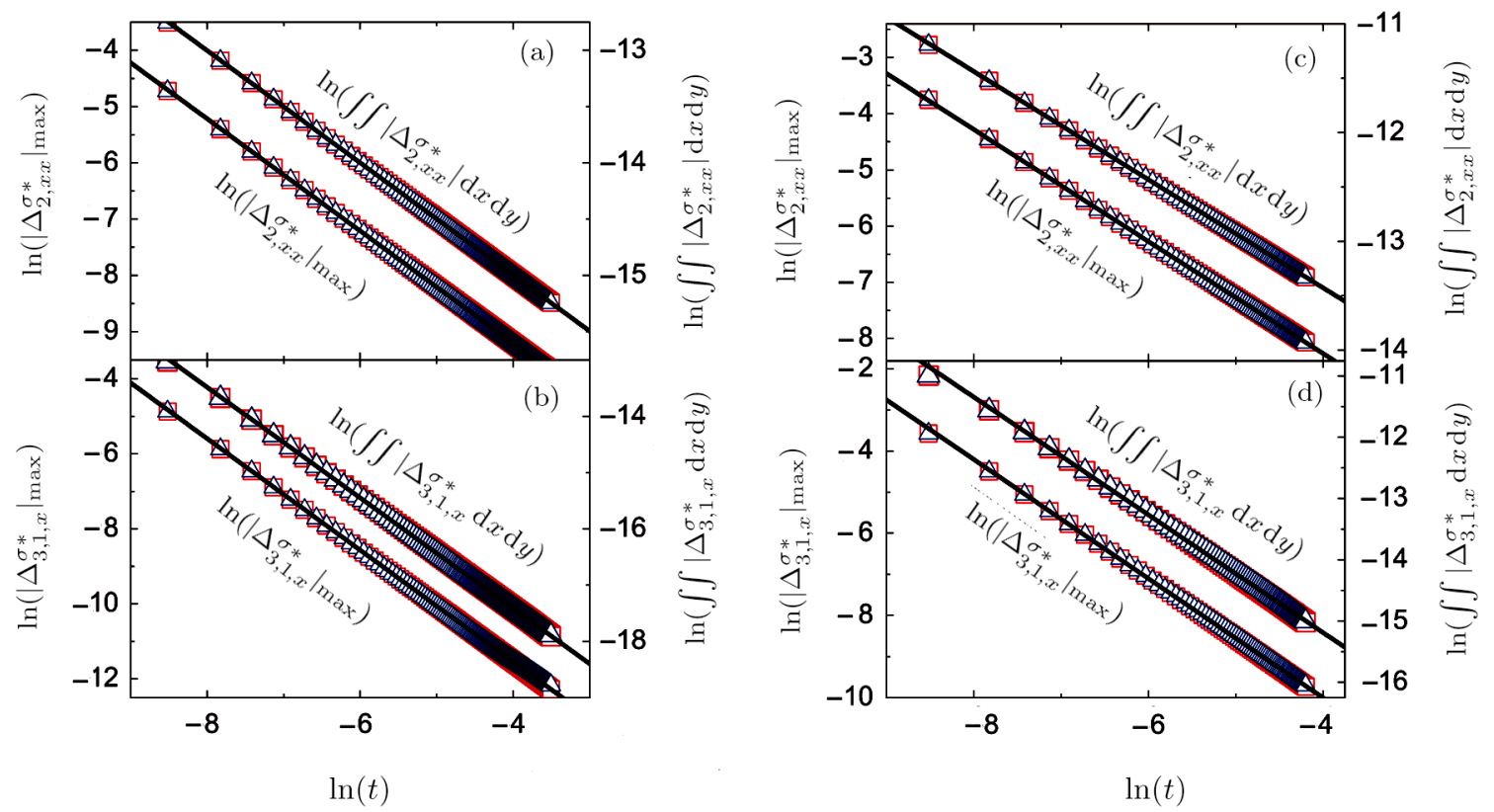

Fig. 3 Evolution of nonequilibrium effects: (a) the amplitude and integral of $\Delta_{2, x x}^{\sigma}$ in Case I, (b) the amplitude and integral of $\Delta_{3,1, x}^{\sigma}$ in Case I, (c) the amplitude and integral of $\Delta_{2, x x}^{\sigma}$ in Case II, (d) the amplitude and integral of $\Delta_{3,1, x}^{\sigma}$ in Case II.
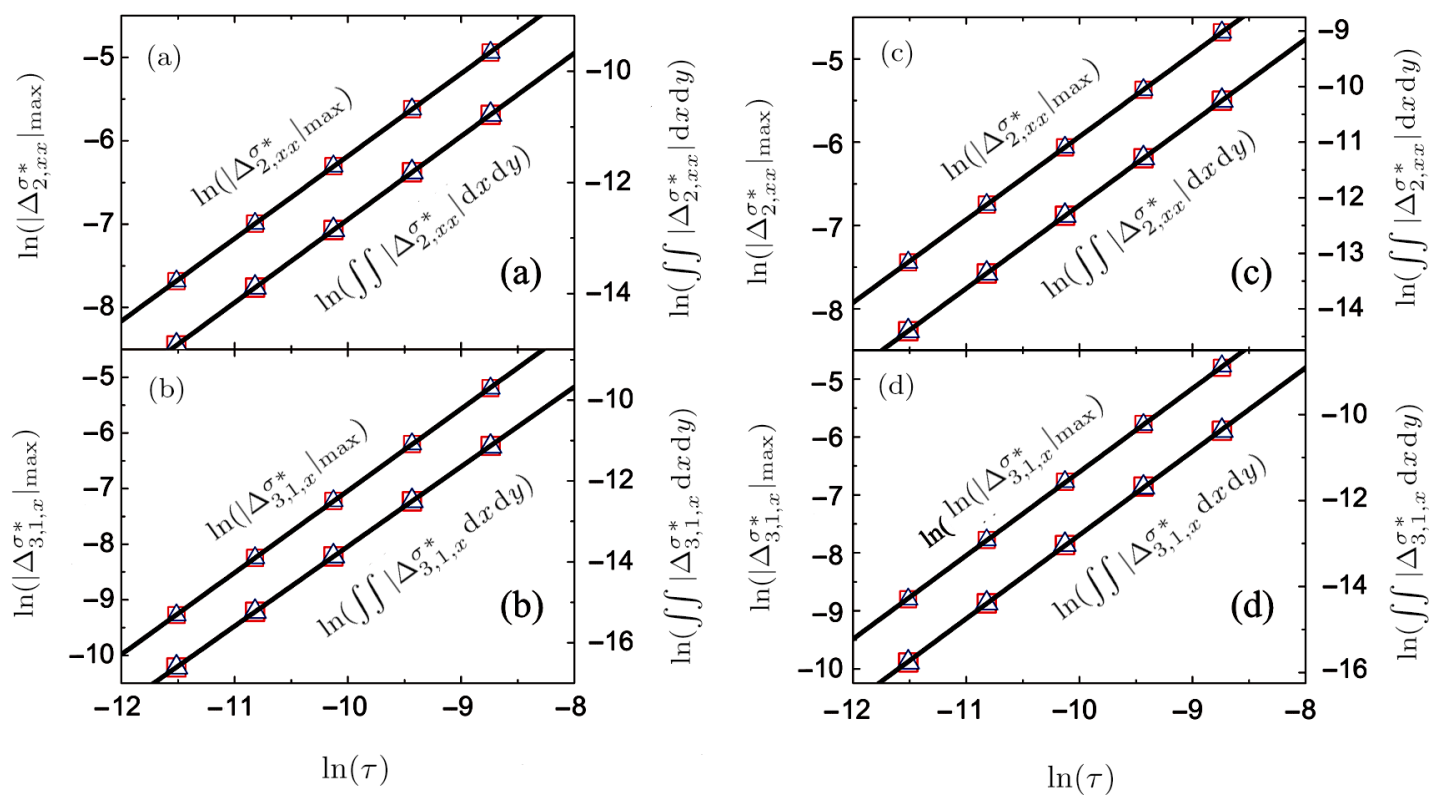

Fig. 4 Nonequilibrium effects versus the relaxation time: (a) the amplitude and integral of $\Delta_{2, x x}^{\sigma}$ in Group I, (b) the amplitude and integral of $\Delta_{3,1, x}^{\sigma}$ in Group I, (c) the amplitude and integral of $\Delta_{2, x x}^{\sigma}$ in Group II, (d) the amplitude and integral of $\Delta_{3,1, x}^{\sigma}$ in Group II. 
In summary, the recently developed discrete Boltzmann method is employed to investigate the TNEs in the dynamic process of binary diffusion. The departure of the velocity distribution function from its equilibrium counterpart is investigated in detail. It is found that, both the local and global TNEs around the material interface decrease with evolution due to the smoothness of physical quantities; while they are enhanced by the relaxation time. Specifically, the relations between the TNEs and the time (the relaxation time) obey a negative (positive) power law.

\section{References}

[1] S. R. De Groot and P. Mazur, Non-Equilibrium Thermodynamics, Courier Corporation, ? (2013).

[2] S. Chapman and T. G. Cowling, The Mathematical Theory of Non-Uniform Gases: an Account of the Kinetic Theory of Viscosity, Thermal Conduction and Diffusion in Gases, Cambridge University Press, ? (1970).

[3] X. Shan and G. Doolen, Phys. Rev. E 54 (1996) 3614.

[4] A. J. Briant and J. M. Yeomans, Phys. Rev. E 69 (2004) 031603.

[5] Z. Chai, C. Huang, B. Shi, and Z. Guo, Int. J. Heat Mass Transf. 98 (2016) 687.

[6] A. S. Joshi, A. A. Peracchio, K. N. Grew, and W. K. Chiu, J. Phys. D: Appl. Phys. 40 (2007) 7593.

[7] F. M. Liu, A. L. Wang, R. F. Qiu, and T. Jiang, Int. J. Mod. Phys. C 27 (2016) 1650130.

[8] S. Hosseini, A. Eshghinejadfard, N. Darabiha, and D. Thévenin, Comput. Math. with Appl. (2017).

[9] J. Zudrop, K. Masilamani, S. Roller, and P. Asinari, Computers \& Fluids 153 (2017) 20.

[10] J. G. Zhou, P. M. Haygarth, P. J. A. Withers, et al., Phys. Rev. E 93 (2016) 043310.
[11] S. Succi, The lattice Boltzmann Equation for Fluid Dynamics and Beyond, Oxford University Press, New York (2001).

[12] R. Qin and Y. Zhang, Comput. Fluids 35 (2006) 929.

[13] C. Zhuo and C. Zhong, Int. J. Heat Fluid Flow 42 (2013) 10.

[14] Y. Gan, A. Xu, G. Zhang, and Y. Li, Commun. Theor. Phys. 57 (2012) 681.

[15] Y. Gan, A. Xu, G. Zhang, and S. Succi, Soft Matter 11 (2015) 5336.

[16] C. Lin, A. Xu, G. Zhang, et al., Phys. Rev. E 89 (2014) 013307.

[17] C. Lin, A. Xu, G. Zhang, and Y. Li, Combust. and Flame 164 (2016) 137.

[18] C. Lin, K. H. Luo, L. Fei, and S. Succi, Sci. Rep. 7 (2017) 14580 .

[19] M. Watari and M. Tsutahara, Phys. Rev. E 67 (2003) 036306.

[20] H. Zhang and F. Zhuang, Adv. Appl. Mech. 29 (1991) 193. 